We have presented the Graphical Abstract text and image for your article below. This brief summary of your work will appear in the contents pages of the issue in which your article appears.

1

\title{
Experimental evidence of charged domain walls in lead-free ferroelectric ceramics: light-driven nanodomain switching
}

Fernando Rubio-Marcos, * Adolfo Del Campo, Rocío E. Rojas-Hernandez, Mariola O. Ramírez, Rodrigo Parra, Rodrigo U. Ichikawa, Leandro A. Ramajo, Luisa E. Bausá and Jose F. Fernández

Emergent phenomena driven by light-matter interaction may have potential applications in next-generation domain wall nanoelectronics utilizing polycrystalline ferroelectrics.

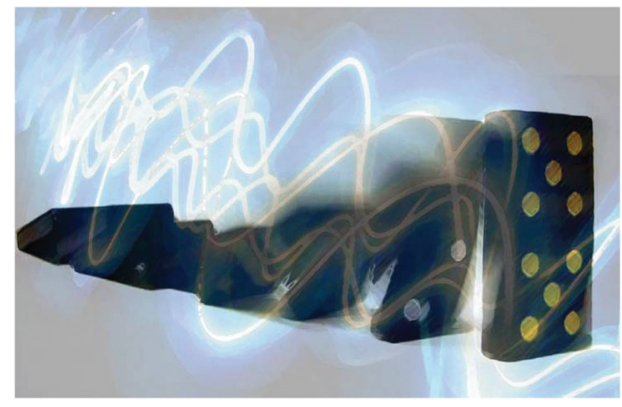

Please check this proof carefully. Our staff will not read it in detail after you have returned it.

Proof corrections must be returned as a single set of corrections, approved by all co-authors. No further corrections can be made after you have submitted your proof corrections as we will publish your article online as soon as possible after they are received.

Please ensure that:

- The spelling and format of all author names and affiliations are checked carefully. Names will be indexed and cited as shown on the proof, so these must be correct.

- Any funding bodies have been acknowledged appropriately.

- All of the editor's queries are answered.

- Any necessary attachments, such as updated images or ESI files, are provided.

Translation errors between word-processor files and typesetting systems can occur so the whole proof needs to be read. Please pay particular attention to: tables; equations; numerical data; figures and graphics; and references.

Please send your corrections preferably as a copy of the proof PDF with electronic notes attached or alternatively as a list of corrections - do not change the text within the PDF file or send a revised manuscript. Corrections at this stage should be minor and not involve extensive changes.

Please return your final corrections, where possible within $\mathbf{4 8}$ hours of receipt, by e-mail to: nanoscale@ arsc.org. If you require more time, please notify us by email. 


\section{Funder information}

Providing accurate funding information will enable us to help you comply with your funders' reporting mandates. Clear acknowledgement of funder support is an important consideration in funding evaluation and can increase your chances of securing funding in the future. We work closely with Crossref to make your research discoverable through the Funding Data search tool (http://search.crossref.org/fundref).

Further information on how to acknowledge your funders can be found on our webpage (http://rsc.li/ funding-info).

\section{What is Funding Data?}

Funding Data (http://www.crossref.org/fundingdata/) provides a reliable way to track the impact of the work that funders support. We collect funding information from our authors and match this information to funders listed in the Open Funder Registry. Once an article has been matched to its funders, it is discoverable through Crossref's search interface.

\section{PubMed Central}

Accurate funder information will also help us identify articles that are mandated to be deposited in PubMed Central (PMC) and deposit these on your behalf.

\section{Providing funder information}

We have included the funder information you gave us on submission in the table below. The 'Funder name' shown and their associated 'Funder ID' number is written as listed in the Open Funder Registry. Please check that the funder names and grant numbers in the table are correct. The funder information should match your acknowledgements. This table will not be included in your final PDF but we will share the data with Crossref so that your article can be found via the Funding Data search tool.

\begin{tabular}{|l|l|l|}
\hline Funder name & $\begin{array}{l}\text { Funder ID } \\
\text { (from the Open Funder Registry) }\end{array}$ & $\begin{array}{l}\text { Award/grant/contract } \\
\text { number }\end{array}$ \\
\hline Consejo Superior de Investigaciones Científicas & 501100003339 & NANOMIND CSIC 201560E068 \\
\hline Ministerio de Economía y Competitividad & 501100003329 & $\begin{array}{l}\text { MAT2013-43301-R } \\
\text { MAT2013-48009-C4-1-P } \\
\text { MAT2016-76106-R } \\
\text { Ramon y Cajal' contract (ref: } \\
\text { RyC-2015-18626) }\end{array}$ \\
\hline
\end{tabular}

If a funding organisation you included on submission of your article is not currently listed in the registry it will not appear in the table above. We can only deposit data if funders are already listed in the Open Funder Registry, but we will pass all funding information on to Crossref so that additional funders can be included in future.

\section{Researcher information}

If any authors have ORCID or ResearcherID details that are not listed below, please provide these with your proof corrections. Please check that the ORCID and ResearcherID details listed below have been assigned to the correct author. Please use this space to add your own unique ORCID iDs and not another researcher's, as errors will delay publication.

Please also update your account on our online manuscript submission system to add your ORCID details, which will then be automatically included in all future submissions. See here for step-by-step instructions and more information on author identifiers.

\begin{tabular}{|l|l|l|l|}
\hline First (given) name(s) & Last (family) name(s) & ResearcherID & ORCID \\
\hline Fernando & Rubio-Marcos & & 0000-0002-2479-3792 \\
\hline Adolfo & Del Campo & & \\
\hline Rocío E. & Rojas-Hernandez & & \\
\hline Mariola O. & Ramírez & $1-3439-2016$ & $0000-0002-1233-1769$ \\
\hline Rodrigo & Parra & &
\end{tabular}




\begin{tabular}{|l|l|l|l|}
\hline Rodrigo U. & Ichikawa & & \\
\hline Leandro A. & Ramajo & & \\
\hline Luisa E. & Bausá & & $0000-0002-8744-213 X$ \\
\hline Jose F. & Fernández & & \\
\hline
\end{tabular}




\section{Queries for the attention of the authors}

Journal: Nanoscale Paper: c7nr04304j

Title: Experimental evidence of charged domain walls in lead-free ferroelectric ceramics: light-driven nanodomain switching

For your information: You can cite this article before you receive notification of the page numbers by using the following format: (authors), Nanoscale, (year), DOI: 10.1039/c7nr04304j.

Editor's queries are marked like this [Q1, Q2, ...], and for your convenience line numbers are indicated like this $[5,10,15, \ldots]$.

Please ensure that all queries are answered when returning your proof corrections so that publication of your article is not delayed.

\begin{tabular}{|c|c|c|}
\hline $\begin{array}{l}\text { Query } \\
\text { Reference }\end{array}$ & Query & Remarks \\
\hline Q1 & $\begin{array}{l}\text { Please confirm that the spelling and format of all author names } \\
\text { is correct. Names will be indexed and cited as shown on the } \\
\text { proof, so these must be correct. No late corrections can be } \\
\text { made. }\end{array}$ & \\
\hline Q2 & $\begin{array}{l}\text { Please check that the Graphical Abstract text fits within the } \\
\text { allocated space indicated on the front page of the proof. If the } \\
\text { entry does not fit between the two horizontal lines, then please } \\
\text { trim the text and/or the title. }\end{array}$ & \\
\hline Q3 & $\begin{array}{l}\text { In the sentence beginning "To date, many of the...", a word or } \\
\text { phrase appears to be missing after "nanoscale". Please check } \\
\text { this carefully and indicate any changes required here. }\end{array}$ & \\
\hline Q4 & $\begin{array}{l}\text { The caption to Fig. } 6 \text { refers to part (e) but the image as supplied } \\
\text { does not contain a part labelled (e). Would you like to modify } \\
\text { the caption or resupply the artwork (preferably as a TIF file at } \\
600 \text { dots per inch)? }\end{array}$ & \\
\hline Q5 & $\begin{array}{l}\text { In the sentence beginning "The solid line represents...", a part } \\
\text { label appears to be missing after "and } \theta=60^{\circ} . \text {. Please check } \\
\text { this carefully and indicate any changes required here. }\end{array}$ & \\
\hline Q6 & $\begin{array}{l}\text { None of the authors given in the author list have the initials } \\
\text { "M. L. B.". Please check this and indicate any changes that are } \\
\text { required here. }\end{array}$ & \\
\hline Q7 & Ref. 20: Please provide the title. & \\
\hline Q8 & Please indicate where ref. 34 should be cited in the text. & \\
\hline
\end{tabular}




\title{
Experimental evidence of charged domain walls in lead-free ferroelectric ceramics: light-driven nanodomain switching $\dagger$
}

\author{
Fernando Rubio-Marcos, (D)*a Adolfo Del Campo, ${ }^{a}$ Rocío E. Rojas-Hernandez, ${ }^{a, b}$ \\ Mariola O. Ramírez, (D) ${ }^{\mathrm{c}}$ Rodrigo Parra, ${ }^{\mathrm{d}}$ Rodrigo U. Ichikawa, ${ }^{\mathrm{e}}$ Leandro A. Ramajo, ${ }^{d}$ \\ Luisa E. Bausá (D) ${ }^{c}$ and Jose F. Fernández ${ }^{a}$
}

The control of ferroelectric domain walls at the nanometric level leads to novel interfacial properties and functionalities. In particular, the comprehension of charged domain walls, CDWs, lies at the frontier of future nanoelectronic research. Whereas many of the effects have been demonstrated for ideal archetypes, such as single crystals, and/or thin films, a similar control of CDWs on polycrystalline ferroelectrics has not been achieved. Here, we unambiguously show the presence of charged domain walls on a leadfree $(K, \mathrm{Na}) \mathrm{NbO}_{3}$ polycrystalline system. The appearance of CDWs is observed in situ by confocal Raman microscopy and second harmonic generation microscopy. CDWs produce an internal strain gradient within each domain. Specifically, the anisotropic strain develops a crucial piece in the ferroelectric domain switching due to the coupling between the polarization of light and the ferroelectric polarization of the nanodomain in the $(\mathrm{K}, \mathrm{Na}) \mathrm{NbO}_{3}$ ceramic. This effect leads to the tuning of the ferroelectric domain switching by means of the light polarization angle. Our results will help to understand the relevance of charged domain walls on the ferroelectric domain switching process and may facilitate the development of domain wall nanoelectronics by remote light control utilizing polycrystalline ferroelectrics.

Received 22nd June 2017 Accepted 29th November 2017 DOI: $10.1039 / \mathrm{c} 7 \mathrm{nr} 04304 \mathrm{j}$ rsc.li/nanoscale
(DWs) are the interface between adjacent domains, in which their properties can be radically different from those of the domains themselves. ${ }^{2-7,11,12}$ New strategies are constantly being developed in order to find effective methodologies capable of modulating the ferroelectric domain motion. ${ }^{3,4,13-16}$ In fact, recent studies have shown that domain walls themselves possess interesting functionalities (e.g., electronic conductivity $)^{1-4,6,13-16}$ and have the potential for other interesting effects, making them potential candidates for active elements in future nanoelectronics. ${ }^{1-4,6,13-16}$

One of the major limitations in ferroelectric domain switching is the required presence of electrical connections or physical contact in practical applications. Recently, T. Sluka et al. ${ }^{14}$ demonstrated the correlation between the existence of charged domain walls (CDWs) in a $\mathrm{BaTiO}_{3}$ (BTO) ferroelectric single crystal and their enhanced electromechanical properties by electron-gas-like conductivity. The discovery of this motivating behavior and the potential technological applications from these enhanced functionalities raise the need for an efficient method to switch ferroelectric domains. For instance, the light-matter interaction process via photostriction in ferroelectrics can be exploited in photovoltaic devices. ${ }^{17-21}$ Recently, the possibility to move ferroelectric domain walls by means of coherent light has been demonstrated. ${ }^{16}$ In a proof-of principle
Electroceramic Department, Instituto de Cerámica y Vidrio, CSIC, Kelsen 5 , Portugal

${ }^{c}$ Department of Física de Materiales and Instituto Nicolás Cabrera, Universidad Autónoma de Madrid, 28049 Madrid, Spain

${ }^{d}$ Instituto de Investigaciones en Ciencia y Tecnología de Materiales (INTEMA), Av. Juan B Justo 4302 (B7608FDQ), Mar del Plata, Argentina

${ }^{e}$ Instituto de Pesquisas Energéticas e Nucleares, IPEN-CNEN/SP, Butantã, São Paulo, SP, 05508-000, Brazil

$\dagger$ Electronic supplementary information (ESI) available: Crystallographic characterization of KNN ferroelectric ceramic by high-resolution synchrotron X-ray diffraction; microstructural characterization and domain width distribution of KNN ceramic calculated from the grain size measured by FE-SEM. See DOI: 10.1039/c7nr04304j

30


1 experiment we demonstrated that the optical control of ferroelectric domain walls is nevertheless possible in ideal archetypes, such as single crystals. ${ }^{16}$ The transfer of this concept to ferroelectric ceramic materials is appealing because of the increasing technological interest in new cost-effective optoelectronic nanodevices.

Manipulating ferroelectric domains for nanoelectronic engineering has been a hot research topic that has aroused great interest. To date, many of the effects observed in novel nanomaterials and nanotechnology have been restricted to ideal archetypes, such as single crystals, and/or thin films (or nanoscale) on which there is a high degree of control over their structure, morphology and geometry. However, the latent role of nanoscale conductivity, particularly at the DWs, on the domain wall motion of polycrystalline ferroelectrics with randomly oriented grains has not been given sufficient attention so far. Indeed, despite the well-developed TEM and PFM experiments to study the domain structure and switching in polycrystals, there are few studies devoted to the occurrence of charged DWs in ferroelectric ceramics. Nonetheless, these polycrystalline materials have important technological implications since they are used in the majority of piezoelectric mechanisms because they are easy to manufacture at a low cost. In these systems even if the local conductivity within the DWs does average macroscopically to a small value, the dynamics of the DWs may still be strongly affected by their conductivity. This effect would be relevant since the domain wall motion shows a major influence on the dielectric and piezoelectric properties of ferroelectric materials, contributing greatly to the macroscopic response. ${ }^{22-25}$ Therefore, the local conductivity at the DWs may meddle with the domain wall switching and, thus, indirectly influence the macroscopic response of polycrystalline ferroelectrics.

Here, we explore the relationship between the strain gradient within the ferroelectric domains and the uncompensated accumulated charge in the domain walls by investigating the photo-induced ferroelectric domain switching in a polycrystalline sample of ferroelectric potassium-sodium niobate $\left(\mathrm{K}_{0.1} \mathrm{Na}_{0.9} \mathrm{NbO}_{3} ; \mathrm{KNN}\right)$ having a monoclinic symmetry. We have stabilized a monophasic state with a domain structure consistent with an $\mathbf{M}_{\mathrm{C}}$ monoclinic phase (space group Pm) (ESI $\left.1 \dagger\right){ }^{26}$ So, the low-symmetry monoclinic phase contributes to the accommodation of the large elastic forces that develop at the morphotropic phase boundary (MPB), and provides elastic matching at the internal interfaces inducing large piezoelectricity. $^{27}$ We find that this strain is anisotropic in ferroelectric domains, and consequently, forms exotic dipole-charged domain walls. We also show that this strain gradient can be controlled by the angle of illumination with a polarized light. Ultimately, the methodology of the present study leads to a non-invasive (no damages are produced at the surface of the ferroelectric material) and reversible process in ferroelectric domain switching. The emergent phenomena driven by lightmatter interaction may have potential applications in nextgeneration domain wall nanoelectronics utilizing polycrystalline ferroelectrics.

\section{Results and discussion}

\subsection{Identification of the domain structure by confocal Raman microscopy}

The presence of crystalline phases plays a key role in the functional properties of ferroelectric ceramics, and therefore, their distribution and identification are relevant to tailor new approaches in designing new materials with improved properties. A standard identification of the orientation and crystalline structure of the ceramic sample was carried out by means of synchrotron X-ray diffraction (HR-XRD) (see ESI Fig. $\mathrm{S} 1$ and Table $1 \dagger)$. The ceramic sample exhibited a monoclinic symmetry $\left(\mathrm{M}_{\mathrm{C}}\right)$ showing two different orientations, $(00 l)$ or c-plane and (h00) or a-plane.

Fig. 1a shows an optical micrograph of the ceramic aligned perpendicular to the Raman laser. The area of $25 \times 25 \mu \mathrm{m}$ denotes the selected spatial region indicated as $\mathbf{1}$ in panel a, where the Raman spectra are recorded at a plane located just below the surface of the sample where the Raman intensity shows a maximum. The acquisition time for a single Raman spectrum was 200 milliseconds; thus the acquisition of a Raman image consisting of $100 \times 100$ pixels (10000 spectra) required 33 minutes. Features such as Raman peak intensity, peak width or Raman shift from the recorded Raman spectra were fitted with algorithms to compare the information and to represent the derived Raman image (Fig. 1b). The $\mathrm{BO}_{6}$ octahedron gives rise to 6 Raman-active modes, $\mathrm{A}_{1 \mathrm{~g}}\left(\nu_{1}\right)+\mathrm{E}_{\mathrm{g}}\left(\nu_{2}\right)+$ $\mathrm{F}_{1 \mathrm{u}}\left(\nu_{3}\right)$ being the stretching modes, and $\mathrm{F}_{1 \mathrm{u}}\left(\nu_{4}\right)+1 \mathrm{~F}_{2 \mathrm{~g}}\left(\nu_{5}\right)+$ $1 \mathrm{~F}_{2 \mathrm{u}}\left(\nu_{6}\right)$ the bending modes. ${ }^{28-30}$ Due to the near-perfect equilateral octahedral symmetry, $\mathrm{A}_{1 \mathrm{~g}}$ and $\mathrm{F}_{2 \mathrm{~g}}$ are dominant in systems similar to the one of this work.

The Raman spectra having the same Raman shift are classified by correlating the color intensity with the Raman intensity. The color assignation to each pixel results in a Raman image of the ceramic microstructure, which reveals the presence of a ceramic grain with striped ferroelectric domains. Regarding the morphology, three types of domains can be recognized in KNN-based ceramics: lamellar-like, needle-like, and irregularly shaped (labelled in Fig. 1b as La Ds, Ne Ds, and Ir Ds, respectively). In the Raman image of Fig. 1b, a striped $90^{\circ}$ domain structure, already reported for $\mathrm{BaTiO}_{3}$ single crystals $^{31}$ and PZT ceramics, ${ }^{32}$ is clearly seen. In addition, a needle-like domain structure is observed, the needlepoints terminating at the grain boundary. This type of domain structure has been previously reported for $\mathrm{PbTiO}_{3}$ thin films, ${ }^{33}$ and bismuth titanate based (BiT) single crystals, and prophesied by Li et al. ${ }^{35}$ The most interesting domain structure (that is, the needle-like domains) is basically constituted of $\sim 400 \mathrm{~nm}$ width domains, which appear alternately (see the area marked on the yellow box and indicted as 3 in panels a and b). Through a classical approach for a polycrystalline sample (ceramic), one can establish that the domain width, $d$, can be roughly expressed by the relation: $d \sim \sqrt{ } a$ (that is, the domain width, $d$, varies as the square root of the grain size, $a),{ }^{6,36}$ which implies that for grain sizes $a \sim 6.7 \mu \mathrm{m}$ (our case), the $d$ value should be $c a$. $2.5 \mu \mathrm{m}$ (ESI $2 \dagger$ ). However, as we just 

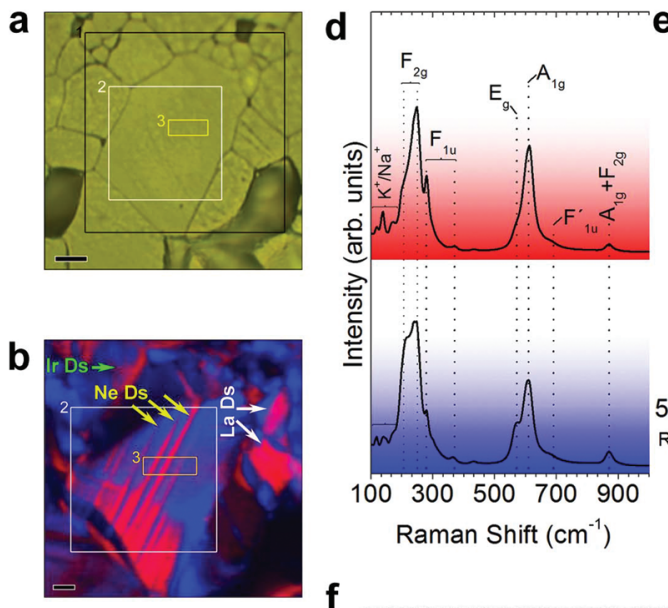

100300500700900

Raman Shift $\left(\mathrm{cm}^{-1}\right)$

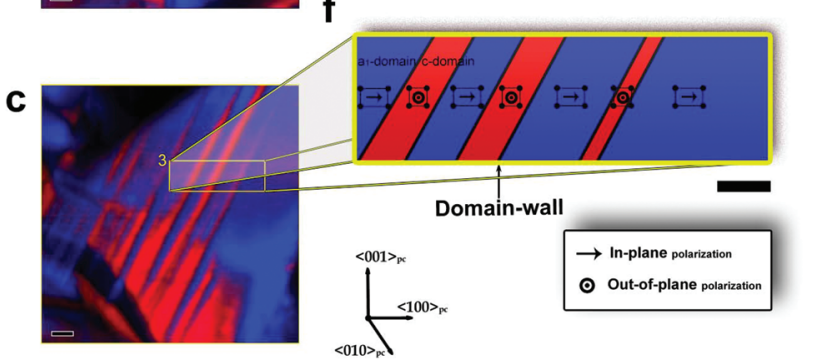

Fig. 1 Identification of the domain structure of a KNN ferroelectric ceramic by Raman imaging. (a) Optical image of the surface of a $(\mathrm{KNa})$ $\mathrm{NbO}_{3}$-based polycrystalline ferroelectric. Scale bar: $4 \mu \mathrm{m}$. The regions marked as rectangles, and indicated as 1, 2 and 3, show the selected areas for XY Raman images. (b) and (c) Surface Raman images resulting from the mapping of the different single Raman spectra obtained from each pixel of the areas labelled as 1 and 2 in (a). The magnification increases from panel b to panel $c$ so that scale bars in (b) correspond to $2 \mu \mathrm{m}$, and in (c) to $1 \mu \mathrm{m}$. In relation to their morphology, the domains can be categorized into different types of domains (Ds) denominated as lamellar, needle, and irregularly shaped, which are marked in panel $b$ as La Ds, Ne Ds, and Ir Ds, respectively. Raman spectra showing the same spectral shift for the Raman modes are identified under the same color intensity. The average Raman spectra of the KNN ceramic image associated with different colours can be observed in panel d. (e) Amplified Raman spectra fitted by the sum of three Lorentzian peaks, ascribed to the $E_{g}, A_{1 g}$ and $F_{1 u}^{\prime}$ Raman modes. It should be taken into account that both $a$ - and $c$-domains are easily evidenced by the $A_{1 g} / E_{g}$ ratio. ${ }^{28}$ Accordingly, panel $d$ allows the identification of the c-domain, i.e. outof-plane polarization (red spectrum), and the identification of the a-domain, i.e. in-plane polarization (blue spectrum). (f) Schematic topview summary of the in-plane and out-of-plane polarization regions as determined by confocal Raman microscopy. Region marked as 3 in panels a-c. The sketch shows a domain structure constituted of a-domain and c-domain separated by a $90^{\circ}$ domain wall, which are represented in blue and red colors, respectively. Scale bar: $500 \mathrm{~nm}$.

evidenced, the relevant domain structure is formed by domains with $d$ values $(\sim 400 \mathrm{~nm})$ significantly smaller than $\sqrt{ } a$ because of its boundary conditions, which are governed by a high internal stress. Generally, the domain size is determined by the competition between the energy of the domains and the energy of the domain walls. ${ }^{6}$ It is worth noting here that the presence of a high stress is addressed by enlarging the domain wall density, resulting in a drastic reduction of the domain size as seen in Fig. 1c. This figure shows a magnified
Raman spatial map of the domain distribution at the surface scan, which corresponds to the area marked on the white box and indicted as $\mathbf{2}$ in panels a and b. The Raman imaging shows that there are significant differences associated with the polarization orientation of each domain compatible with alternating in-plane and out-of-plane domains, and as a result of the existence of $90^{\circ}$ domain walls between adjacent needle-like domains. For an in-plane polarized a-domain, the doublet of the $\mathrm{BO}_{6}$ octahedron stretching $\mathrm{E}_{\mathrm{g}}\left(\nu_{2}\right)$ and $\mathrm{A}_{1 \mathrm{~g}}\left(\nu_{1}\right)$ Raman modes can be observed for Raman shifts between 500-700 $\mathrm{cm}^{-1}$, while an out-of-plane polarized c-domain should have only one Raman mode corresponding to the $\mathrm{A}_{1 \mathrm{~g}}$ $\left(\nu_{1}\right)$ mode in this range. The experimental data further confirm the polarization orientation of each domain, as shown in Fig. 1d and e. In this case, as occurs in complex systems such as ceramic materials, a small contribution of the $\mathrm{E}_{\mathrm{g}}\left(\nu_{2}\right)$ Raman mode is observed on out-of-plane polarized c-domains due to a slight tilting of the $\mathrm{BO}_{6}$ octahedron of the perovskite structure. See Fig. 1e.

In short, CRM reveals that $(\mathrm{K}, \mathrm{Na}) \mathrm{NbO}_{3}$-based ceramics exhibit an a/c/a/c-like domain structure, which is constituted of in-plane polarized a-domains (marked in blue color) and out-of-plane polarized c-domains (signaled in red color) separated by a $90^{\circ}$ domain wall, as shown in the scheme of Fig. 1f.

\subsection{Identification of the internal strain gradient within each domain}

To verify again the polarization orientation and to probe the existence of strain gradients in the a/c/a/c-like domain structure, we first focus on specific relevant zones, that is on the domain walls (DWs) and on the inner region of the ferroelectric domains represented in Fig. 2a as adjacent strip areas separated by $90^{\circ}$ DWs. Fig. $2 \mathrm{~b}$ shows the sequential details of the stretching $\mathrm{E}_{\mathrm{g}}\left(\nu_{2}\right)$ and $\mathrm{A}_{1 \mathrm{~g}}\left(\nu_{1}\right)$ Raman modes of the $\mathrm{BO}_{6}$ octahedron along the different points marked in Fig. 2a. As has been anticipated, the $\mathrm{A}_{1 \mathrm{~g}} / \mathrm{E}_{\mathrm{g}}$ ratio can be used to define the polarization orientation of the ferroelectric domain structure. That is, the $A_{1 g} / E_{g}$ ratio shows a maximum/minimum value when the coherent light polarization is perpendicular/parallel to the ferroelectric polarization, which according to our experimental configuration corresponds to the out-of-plane polarized c-domains/in-plane polarized a-domains, respectively. The evolution of the $A_{1 g} / E_{g}$ ratio is plotted in Fig. 2c.

With regard to DWs, their nature cannot be evidenced by means of the $A_{1 g} / E_{g}$ ratio and therefore, we need to use other relevant features as the Raman shifts that evidence chemical bond variations of the $\mathrm{BO}_{6}$ octahedra related to the crystalline strain. Here, we use the Raman shift of the $A_{1 g}$ mode that corresponds to a symmetric vibration of the oxygen octahedra to determine the nature of the DWs. Fig. $2 \mathrm{~d}$ shows the Raman shift of the $\mathrm{A}_{1 \mathrm{~g}}$ mode along the ferroelectric domain structure, which provides the local strain evolution with nanometric resolution. As observed, the Raman shift, and therefore the local strain, shows a clear oscillating trend along the a/c/ a/c-like domain structure, their maximum and minimum values being located close to the DWs. The alternating maxima 
a
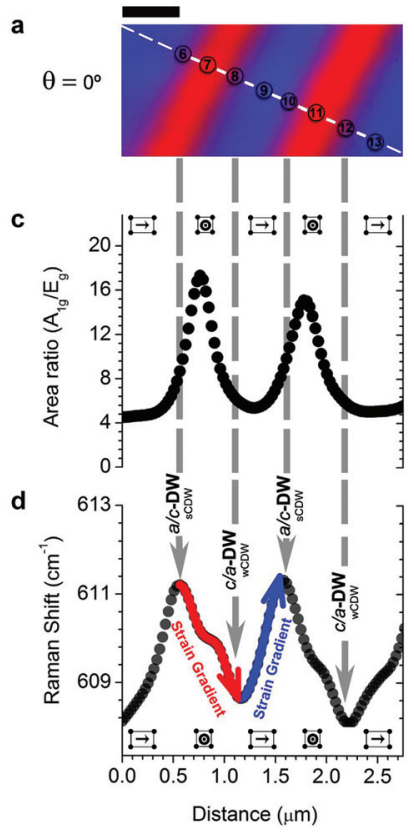
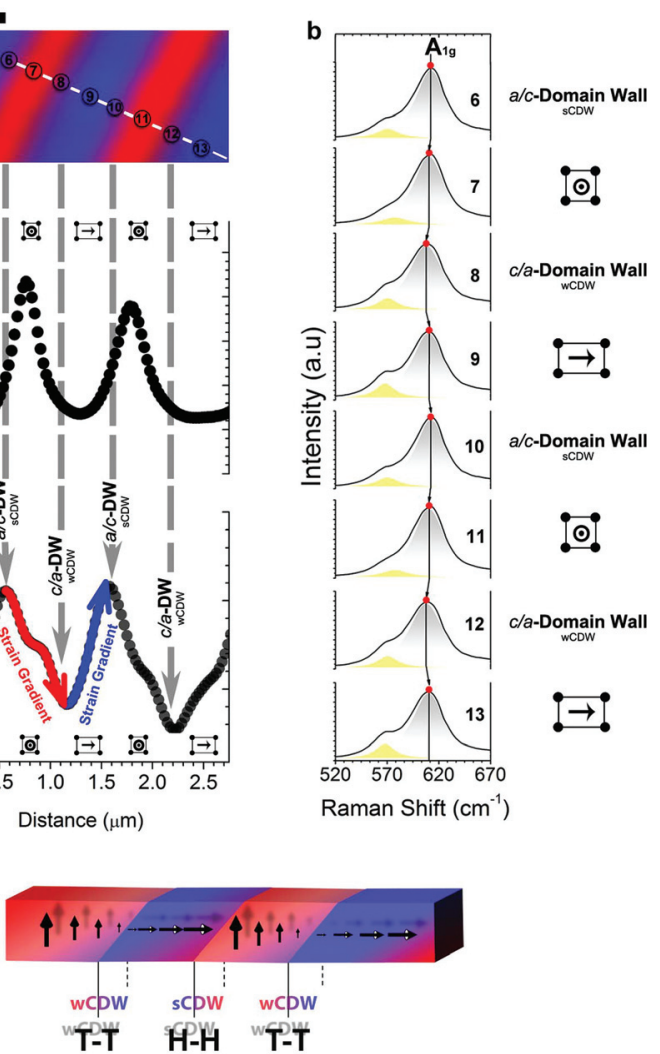

and minima in the Raman shift of $\mathrm{A}_{1 g}$ across the domain structure are related to a/c and c/a DWs, respectively. The a/c DWs are characterized by a Raman blueshift associated with a higher force constant for the oxygen octahedra. Meanwhile, the c/a DWs show a Raman redshift that accounts for a lower force constant of the oxygen octahedra. Accordingly, the presence of a strain gradient in the domain structure is demonstrated.

The presence of strain gradients evidences the presence of DWs which are not electrostatically neutral. The DWs located at both maximum and minimum strain values are special cases of the violation of the electrostatic neutrality, ${ }^{37}$ resulting in the formation of "charged domain walls" (CDWs). These singular CDWs have been demonstrated in both thin films ${ }^{38-40}$ and single crystals, ${ }^{14}$ and recently in KNN ceramics ${ }^{41}$ - the subject of this study. Moreover, the mechanical writing of charged conducting walls through a locally applied point stress has been recently reported in $\mathrm{Cu}_{3} \mathrm{~B}_{7} \mathrm{O}_{13} \mathrm{Cl}$ single crystals. $^{42}$

The DWs located at the maximum strain values should be categorized into a type of CDW denominated as strongly charged domain walls (sCDWs), typically having a head-tohead configuration, while those DWs detected at low strain values can be classified as weakly charged domain walls (wCDWs), typically of the tail-to-tail configuration. ${ }^{43}$ The effect of CDWs on the Raman shift of the $\mathrm{A}_{1 \mathrm{~g}}$ mode is quite relevant. The out-of-plane polarization regions show higher force constants indicating a higher displacement of the $\mathrm{Nb}^{5+}$ cation from the electrostatic equilibrium position in the oxygen octahedra. Thus, the larger the Raman shifts, the higher the piezoelectric response..$^{30}$ On the contrary, the in-plane polarization regions show Raman redshift due to strain constrictions. The presence of CDWs enhances the octahedral distortion, and hence locally contributes to the Raman blueshift; meanwhile, the wCDWs reduce the polarization. The strain gradient across the alternate needle domains results in a combination of outof-plane and in-plane polarization regions with the modulation of CDWs (see ESI S $3 \dagger$ ).

So far, we have demonstrated the existence of strain gradients in an $\mathrm{a} / \mathrm{c} / \mathrm{a} / \mathrm{c}$-like domain structure transiting from maximum to minimum strain states, which are localized at the $\mathrm{a} / \mathrm{c}$ and c/a domain walls, respectively (see the schematic illustration of Fig. 2e). In addition, a correlation between $\mathrm{BO}_{6}$ distortion and the remnant polarization $\left(P_{\mathrm{r}}\right)$ in KNN-based ceramics has been shown by studying the Raman spectroscopy. ${ }^{30}$ From the linear relationship existing between the $\mathrm{A}_{1 \mathrm{~g}}$ Raman mode shift and $P_{\mathrm{r}}{ }^{30}$ we infer that the observed shift of $4 \mathrm{~cm}^{-1}$ in Fig. $2 \mathrm{~d}$ corresponds to a $P_{\mathrm{r}}$ variation larger than $20 \%$. Thus, we can establish the existence of a polarization gradient increasing at the a-c-DWs. ${ }^{44}$ The greater Raman shift is an indirect proof of the electric field enhancement created at the head-to-head (H-H) a-c domain walls (Fig. 2e). Conversely, the tail-to-tail configuration (T-T) at the c-a DWs exhibits the minimum strain state and, consequently, a lower polarizability. In accordance with the observations of Gao et al.,${ }^{45}$ the a-c domain wall is a transition zone of mixed polarization, in which the a-domain (at the left of the a/c domain wall) shows a higher polarization, transiting to the minimum polarization state along the c-domain. In other words, this means that the polarization transition zone is located close to the a/c domain wall whose role is to minimize the charge density. This latter 
configuration perfectly agrees with the conductivity behavior recently predicted by Setter's group ${ }^{43}$ (see the schematic illustration in Fig. 2e) as well as with the elastic compatibility conditions between specific domain pairs reported in ref. 42 . Furthermore, the results are consistent with recent studies based on PFM experiments where the presence of CDWs in KNN was experimentally confirmed for large grain sizes (1-10 $\mu \mathrm{m})$ by means of PFM experiments. ${ }^{41}$

On the basis of the above analysis, we can establish a phenomenological set of features that are of great importance to find CDWs in ceramics. Firstly, the CDWs appear in large grains since for small grain sizes ${ }^{41}(<1 \mu \mathrm{m})$, the grain boundary limits the formation of non- $180^{\circ}$ domains. The stress in the ceramics occurs as a consequence of the polymorphic phase transformation in a perovskite unit cell $\mathrm{ABO}_{3}$ from the paraelectric to ferroelectric state during the cooling of the sintering process. The appearance of a polar direction introduced stress in the grains that could not be accommodated by $180^{\circ}$ domains and requires polarization rotation by non- $180^{\circ}$ domains. If the grain size is low enough the grain boundary energy could avoid the formation of such non- $180^{\circ}$ domains. Recently, the observations by Esin et al. ${ }^{41}$ using PFM confirmed that the formation of non- $180^{\circ}$ domains is required for the formation of CDWs. Secondly, the domain wall density is higher than expected for the grain size found in our study. The domain width is smaller than predicted for ceramics (as evidenced in ESI S2 $\dagger$ ). This means that the formation of CDWs serves as a mechanism to accommodate larger stresses than in regular domain structures. And finally, the ceramic in this study possesses a monoclinic symmetry $\left(\mathrm{M}_{\mathrm{C}}\right)$ that allows a higher number of polarization directions, and thus the switching of polarization. $\sim 90^{\circ}$ and $\sim 60^{\circ}$ domain switching could nucleate in the positions of the local stress to release elastic energies and therefore the internal stress in $180^{\circ}$ domain switching could be effectively relaxed by establishing $\sim 90^{\circ}$ and $\sim 60^{\circ}$ domain stripes, as evidenced by Deng et al. ${ }^{46}$ The domains in the presence of CDWs show a structural gradient that supports a polarization gradient, as evidenced in Fig. 2.

\subsection{Imaging and analysis of the ferroelectric domain structure by second harmonic generation microscopy}
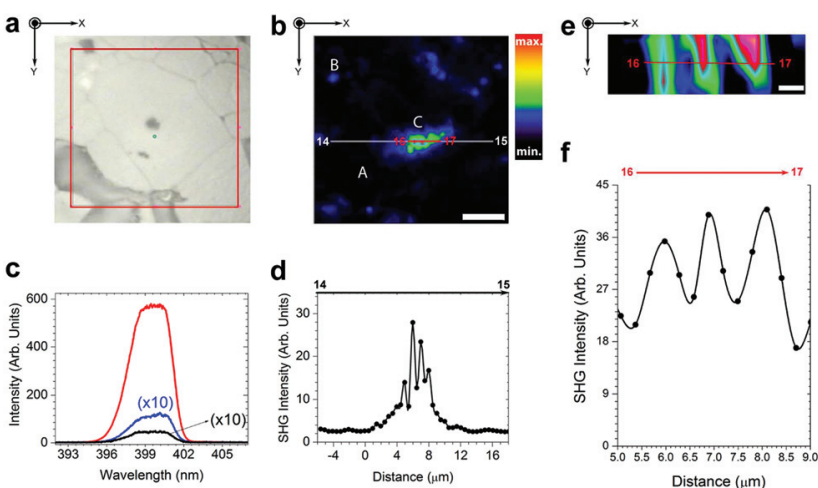

Fig. 3 Imaging and analysis of the ferroelectric domain structure in KNN by second harmonic generation microscopy. (a) Optical micrograph of the spatial area scanned by SHG. (b) SHG microscopy image displaying the in-plane (black color) and out-of-plane (blue color) ferroelectric domain structure present in the KNN ceramic. The contrast in the image arises from the different SHG intensity values obtained when different tensor components (nonlinear coefficients) are involved in the nonlinear process. Scale bar: $3 \mu \mathrm{m}$. (c) SHG spectra recorded at the a-domains (black line), c-domains (blue line,) and central region of the grain where the strongest nonlinear response (SHG intensity) was monitored (red line). For the sake of comparison, the SHG spectra recorded at the isolated a/c ferroelectric domains have been multiplied by a factor of $\times 10$. (d) SHG intensity profile obtained by integrating the total emitted area as a function of the spatial position across the line denoted as $14 \rightarrow$ 15 in panel b. (e) Spatially resolved SHG image recorded at the central region of the grain with a larger magnification. The colour code reflects the intensity variations across the striped-like nanodomains. Scale bar: $0.5 \mu \mathrm{m}$. (f) SHG intensity profile obtained across the striped-like ferroelectric domains displayed in panel e. The scanned line has been denoted as $16 \rightarrow 17$.

complementary tool to Raman spectroscopy imaging for unraveling the presence of charged domain walls.

Fig. 3 shows the optical (Fig. 3a) and SHG image (Fig. 3b) obtained when scanning the same spatial region analyzed by Raman spectroscopy. For the experiments, the fundamental beam was polarized parallel to the $x$-axis in the figure. As expected, the spatial map of the SHG intensity reveals the presence of the two types of ferroelectric domains in the sample (black and blue color in the image), their contrast generated by the different nonlinear coefficients (tensor components probed by the incident beam) involved in the SHG process. Additionally, a well-defined area showing a much stronger SHG signal is clearly distinguished at the central region of the image. Fig. 3c depicts the SHG spectra recorded at the three regions of interest: a-domains (black line), c-domains (blue line,) and the central region of the image, where the strongest nonlinear response (SHG intensity) was observed (red line). For illustrative purposes, these regions have been marked in Fig. 3b, by A, B and C. As observed, the SHG intensity obtained at the central region is about one order of magnitude higher than that obtained at the isolated a/c ferroelectric domains. Furthermore, as illustrated in Fig. 3d, the SHG intensity profile across this spatial region is composed of a peak-valley structure with a separation between the maxima of about $1 \mu \mathrm{m}$.

5
ceramic. Furthermore, the superior sensitivity of SHG to subtle changes in the crystal symmetry would also allow the detection of any local inhomogeneous strain in the system, ${ }^{47}$ providing a

(

(1)


To get further insights into this aspect, Fig. 3e shows a close-up view of the SHG scan in the central region. As seen, the periodical spatial modulation of the SHG intensity can be directly related to the formation of ultrafine striped-like ferroelectric domains of about $500 \mathrm{~nm}$ width, in good agreement with the Raman results (Fig. 1). Moreover, as illustrated by the color code in the figure, the SHG intensity across the stripedlike nano-domains is not uniform, but shows a spatial gradient. The oscillating trend of the nonlinear response across the nano-domain structure is further illustrated by the SHG intensity profile shown in Fig. 3f. Note that the SHG signal initially increases at the first nano-domain, then reaches a maximum at the domain wall boundary and decreases in the subsequent nano-domain until reaching the next wall. Such a spatial distribution is consistent with the different strain fields associated with the striped needle-like ferroelectric domains, and indicates that the maximum and minimum values of the gradient are spatially localized at the domain walls.

\subsection{Relevance of charged domain walls in the domain switching mechanism}

A relevant feature of ferroic materials is the possibility of an active control of their functionalities at the nanoscale via external stimuli such as applied stress, magnetic or electric fields, and in this particular case, polarized light. In situ observations of polarization switching were carried out by illuminating with a polarized light. Fig. $4 \mathrm{a}-\mathrm{g}$ present the evolution of the Raman images of the surface of KNN ceramics in which ferroelectric domains shift along the $X$ axis. The $\mathbf{1 8} \rightarrow \mathbf{1 9}$ line serves as a reference guide to follow the domain movement. From Fig. 4a$\mathrm{g}$, one can deduce that the light polarization direction alters the Raman modes. The Raman spectrum of the red domain (i.e. out-of-plane polarized c-domain) evolves toward the blue domain (i.e. in-plane polarized a-domain), which accounts for a structural change.

In order to further determine the strain degree, and consequently, as shown in Fig. 2d, its polarizability degree associated with the domain movement, the Raman shift image of the $\mathrm{A}_{1 \mathrm{~g}}$ Raman mode for each light polarization angle is also shown in Fig. 4h-n. Both the a-domain and c-domain show a maximum Raman shift for a light polarization angle of $60^{\circ}$, coinciding with the DWs. A minimum Raman shift is observed for a light polarization angle of $30^{\circ}$, at the direction perpendicular to the DWs. Moreover, the Raman shift differences between the in-plane and out-of-plane polarization region show a minimum at $-30^{\circ}$ and a maximum at $60^{\circ}$, as a proof of the strain evolution with the angle of polarization of light $(\theta)$. So, the Raman shift changes with the light polarization angle and the DW motion are correlated with the light polarization angle (see Fig. 4h-n).

Fig. 4o shows the influence of the light polarization angle on the final domain position. The relative motion is plotted by using as reference the line marked as $\mathbf{1 8} \rightarrow \mathbf{1 9}$ in Fig. $4 \mathrm{a}-\mathrm{g}$ and $\mathrm{h}-\mathrm{n}$. In this way, we can infer that the relative motion of the
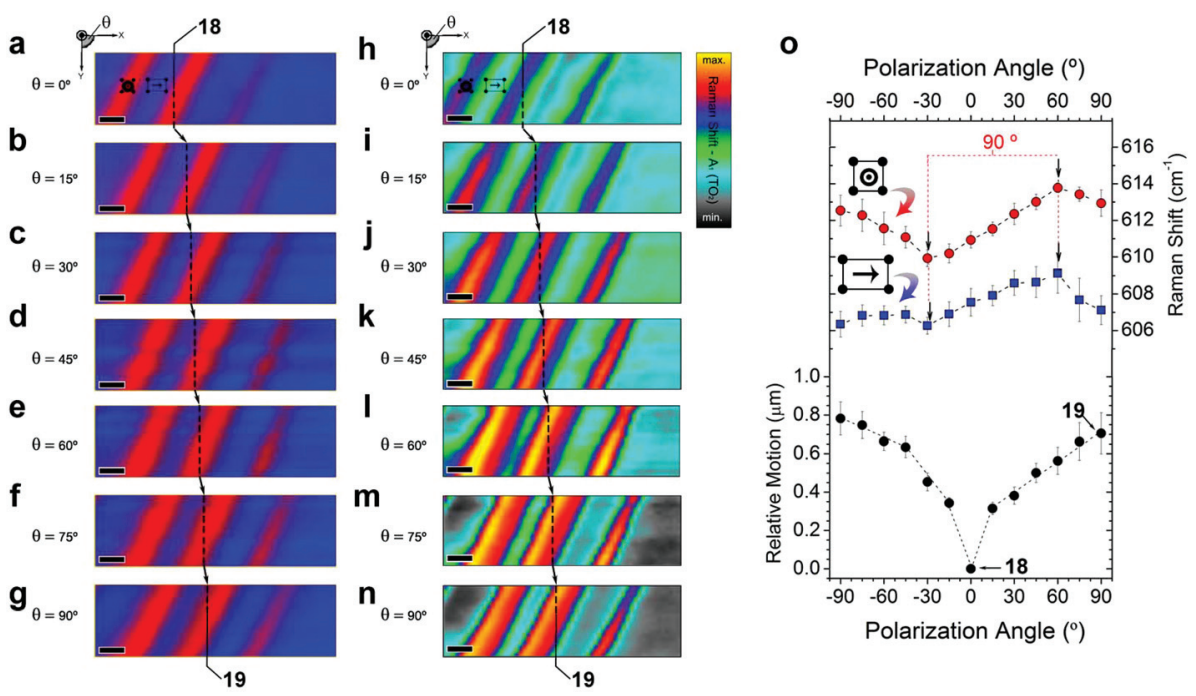

Fig. 4 Light-controlled ferroelectric domain switching in polycrystalline ferroelectric. (a-g) Set of Raman images showing the switching of the domain structure of the KNN surface section (area marked as 3 in Fig. 1c), for light polarization angles between $-90^{\circ} \rightarrow 0^{\circ} \rightarrow 90^{\circ}$. Red regions denote the out-of-plane polarization or c-domain, and blue regions denote the in-plane polarization or a-domain. The scheme at the top of Fig. $4 a$ represents the coordinates where the light polarization angle $\theta$ at the surface of the crystal varies in the plane XY. Additionally, the value of the light polarization angle $\theta$ is indicated on the left side of each Raman image. Scale bar: $500 \mathrm{~nm}$. $(\mathrm{h}-\mathrm{n})$ A collection of Raman shift images showing the strain evolution on the ferroelectric domain structure. The relative motion of domains is illustrated along the line marked as $18 \rightarrow 19$. (o) Evolution of the $A_{1 g}$ Raman shift for two principal adjacent domains (i.e. an in-plane polarization or a-domain, signaled in blue, and an out-of-plane polarization or c-domain, signaled in red) as a function of the light polarization angle. The error bars show the standard deviation (sd) of the Raman shift evolution for three main points in the domain structure for each angle of light polarization on a given sample. The relative change position of the domain is shown along the line marked as $18 \rightarrow 19 \mathrm{in}(\mathrm{h}-\mathrm{n})$, and is also depicted as a function of the polarization angle $(\theta)$ at the bottom of (o). The error bars correspond to the $s d$ of the relative motion. 
domains is approximately $780 \pm 90 \mathrm{~nm}$ when rotating the polarization from 0 to $90^{\circ}$. More specifically, the anisotropic strain (evidenced in Fig. 2d) is correlated with alternating strongly and weakly charged domain walls, playing a key role in the ferroelectric domain switching.

Moreover, the domain switching mechanism is evidenced by the clear evolution of the domain width $(d)$ observed in Fig. 4. A depth analysis of the evolution of $d$ is shown in Fig. 5 . The two relevant cases corresponding to $\theta=0^{\circ}$ and $\theta=60^{\circ}$ are depicted in Fig. 5a and b, respectively. We find a remarkable correlation between the increased $d$ corresponding to out-ofplane polarized c-domains (that is, a dimensional change) and the tuning of the light polarization angle; see c-domain indicated with 20 and $20^{\prime}$ in Fig. $5 \mathrm{a}$ and b. The line scan profile of the phase volume for each domain in Fig. 5a shows an oscillatory behavior, perfectly correlated with the a/c/a/c-like domain structure. It is formed by out-of-plane polarized c-domains (red color) and in-plane polarized a-domains (blue color) separated by a $90^{\circ}$ domain wall (determined by the intersection between the two curves shown in the line profile of Fig. 5a). In Fig. 5c, we plot the relative growth of $d$ for a c-domain (indicated with 20 and $20^{\prime}$ ) as a function of $\theta$. This analysis shows that the $d$ growth is approximately $183 \pm 30 \mathrm{~nm}$ when $\theta$ changes from 0 to $60^{\circ}$. It is worth noting that at $\theta \approx$ $60^{\circ}$ the electric field of the polarized light is nearly parallel to

Fig. 5 Influence of the polarized light on the domain width. (a-b) Spatial map of the Raman signal showing the domain structure at the surface by a colour code, which corresponds to $\theta$ of $0^{\circ}$ and $60^{\circ}$. Scale bar: $500 \mathrm{~nm}$. A line-scanned profile of the relative volume of each ferroelectric domain is plotted at the bottom of each image (indicated by the white dashed arrows). (c) Relative domain width evolutions for the the domain walls. As expected, the adjacent a-domain under- a

b

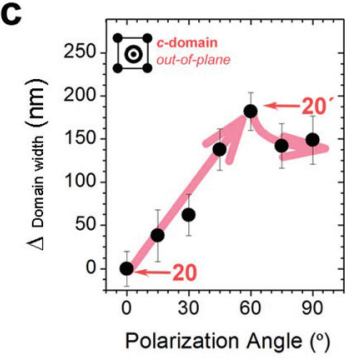

b

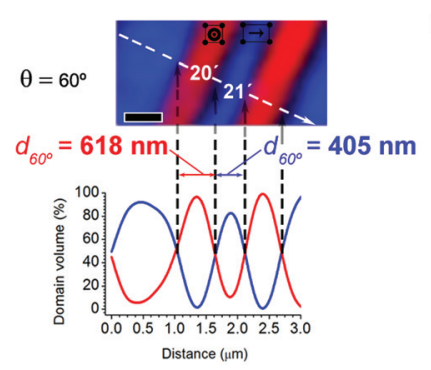

d

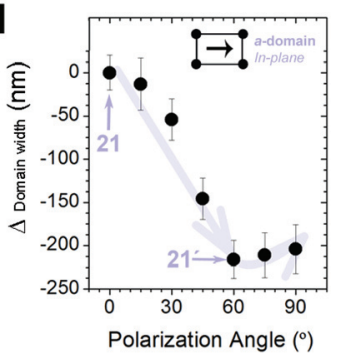
c-domain (indicated with 20 and 20' in panels $a-b$ ) and (d) for the a-domain (signaled with 21 and $21^{\prime}$ in panels a-b) plotted as a function of the polarization angle $\theta$. The error bars show the $s d$ of the relative motion. goes a proportional decrease in $d$, which is indicated with 21 and $21^{\prime}$ in Fig. 5a and b (its relative growth is plotted in blue color in Fig. $5 \mathrm{~d}$ ). The results provide a conclusive proof that a light-induced local electric field is able to perform $90^{\circ}$ domain switching, thereby modifying the domain dimension.

Before concluding, it is worth considering that the effect presented in this work can be explained with the scheme shown in Fig. 6a and b. One of the main aspects to take into account is the slight DW mismatch between the two ferroelectric domains, which triggers the formation of sCDWs. Locally, the formation of sCDWs may vary substantially the net polarization into each domain, resulting in a strain gradient. The formation of strain patterns also affects the light-driven domain switching mechanism. Upon polarized light illumination, non$180^{\circ}$ domain switching occurs and produces a net dimensional change (with respect to the initial state) along the light polarization direction compatible with the orientation of the domains. As the crystal structure is monoclinic, $\mathbf{M}_{\mathrm{C}}$ type, the Ps can rotate in a direction between the tetragonal and the orthorhombic polar crystallographic directions. In particular, the monoclinic phase $\mathrm{M}_{\mathrm{C}}$ is defined by a polarization direction that deviates from any of the 12 orthorhombic polarization

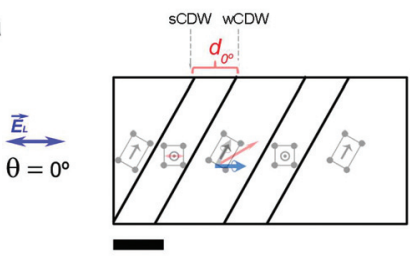

C
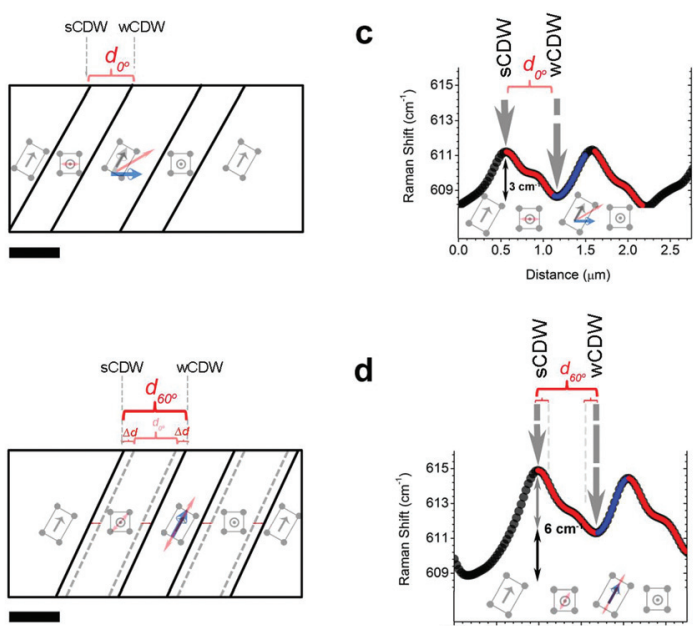

d

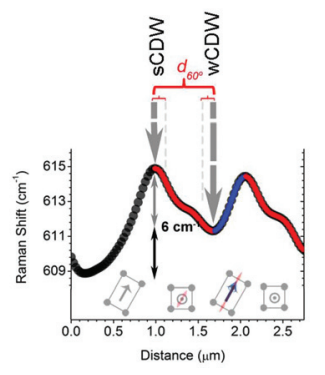

Fig. 6 The effect of charged domain wall on the light-driven domain switching mechanism. (a-b) Schematics of the main contributing polarization directions with their respective polarization vectors for the special cases $\theta=0^{\circ}(\mathrm{a})$ and $\theta=60^{\circ}(\mathrm{b})$. The scale bar for both panels $\mathrm{d}$ and $e$ is $500 \mathrm{~nm}$. The direction of the blue, grey and red arrows depicts the light polarization, polarization vector and the resulting macroscopic polarization, respectively. The solid line represents the DWs at $\theta=0^{\circ}(\mathrm{d})$, and $\theta=60^{\circ}$, while the dashed line shows the relative $d$ growth for a c-domain by means of $\theta$ variation from $\theta=0^{\circ}$ to $\theta=60^{\circ}$. The schematic representation shows a strongly charged wall (sCDW), which is related to a head-to-head $90^{\circ}$ domain wall, whereas the weakly charged domain wall (wCDW) has a tail-to-tail configuration. Besides, the value of $\theta$ and its light polarization direction (marked with a blue double arrow) are indicated on the left side of each scheme. Line-scanned profile of the Raman shift of the $A_{1 g}$ mode for the cases $\theta=0^{\circ}(d)$, and $\theta=60^{\circ}$ (e). Line-scanned profiles correspond to the white dashed arrows of Fig. $5 a$ and b. 


\section{Conclusion} shown in ESI S4.† directions by more than $5^{\circ} .^{48}$ Under the electric field associated with the polarized light, the monoclinic polar directions are able to move in order to relieve the crystal stress imposed by the domain walls. The occurrence of multiple polar directions in the monoclinic phase favors the Ps rotation. The electric field of the polarized light allows enhancing the ferroelectric polarization as it is stated from the blueshift of the Raman mode $A_{1 g}$. It is interesting to point out that the same behaviour is displayed in both Raman shift evolution (Fig. 6c and d) (that is, strain evolution) and relative $d$ growth (Fig. 5c and d) versus the light polarization angle $(\theta)$. In other words, the strain evolution and the relative growth, $d$, first increase and then slightly decrease as $\theta$ increases, reaching a maximum at $\theta=60^{\circ}$. As the domain width increases when the direction of the polarized light is almost parallel to the domain wall, two aspects contribute to the enhancement of the ferroelectric polarization revealed by the Raman blueshift: the stress relief and the enhancement of the CDW effect. Moreover, the variation in the angle of the polarized light with respect to the crystallographic polar directions produces a variation in charge at the CDWs equal to the variation of the electric field. The evolution of polarization with the domain switching provides a conclusive proof that a light-induced local electric field is able to perform $90^{\circ}$ domain switching modifying the domain dimension. Thus, the alignment of the light polarization direction with the domain wall produces a variation in charge at the CDWs similar to a variation of the electric field. A schematic representation showing the effect of electrical field application on a polycrystalline ferroelectric with randomly oriented grains is

Summarizing, the $c$-domain shows a width increase when the polarized light is parallel to the DWs, indicating that the effect of light is similar to the application of an electric field in the out-of-plane polarization direction. In addition, the increase in width of the out-of-plane polarization when the polarized light is parallel to the DWs could only be correlated with the charge variation across the CDWs, and thus the effect of the associated local electric field. The polycrystalline material considered in this work, with imperfect DW matching between the individual domains, is obviously a non-idealized structure. Thus, the analysis of its optical fingerprints presented here is an essential step forward in view of identifying and understanding the features of real systems.

To conclude, utilizing a combination of quantitative CRM with SHG analysis, we have demonstrated the existence of stable CDWs in polycrystalline ceramics by using a prototypic KNNbased material having a monoclinic phase. The CDWs are located in large grains having a larger density of domain walls than that predicted from the grain size rule. ${ }^{36}$ It is shown that the presence of CDWs is not exclusive to exotic systems since they are commonly present in ceramic materials. The ferroelectric domains possess strain gradients as a consequence of highly charged CDWs characteristic of the head-to-head configuration; meanwhile, low charged CDWs correspond to the tail-to-tail configuration. The domain motion of CDWs is attained through the variation of the polarization angle of the incident light. The coupling of polarized light with the ferroelectric structure produced a variation of the crystallographic polar direction that explains the domain motion due to the strain change experimented by the ferroelectric regions. In addition, the alignment of the light polarization direction with the domain wall produces a variation in charge at the CDWs equal to the variation of the electric field. In the present work the domain wall motion has been achieved only by the application of polarized light without electric contacts or external electric field, resulting, thus, in a relevant approach to nanodomain motion control in new optoelectronic nanodevices. It is important to point out that light-driven ferroelectric domain switching can be modulated through the control of the light polarization angle. Moreover, this approach opens new possibilities to understand both the generation of ferroelectric domains and the dynamics of domain motion in piezoelectric ceramic materials. These findings point the way towards more stable device designs based on the large-scale production of ferroelectric materials.

\section{Experimental section}

Sample preparation: For the preparation of the $\mathrm{K}_{0.1} \mathrm{Na}_{0.9} \mathrm{NbO}_{3}$ (KNN) precursor, a $10 \mathrm{M}$ alkaline solution is prepared using potassium hydroxide ( $\mathrm{KOH}, 99.0 \%$, Sigma-Aldrich) and sodium hydroxide (NaOH, 99.5\%, Sigma-Aldrich) at a molar ratio of 7.5:2.5 in DI water. Then, 2 grams of niobium oxide $\left(\mathrm{Nb}_{2} \mathrm{O}_{5}, \quad 99.999 \%\right.$, Sigma-Aldrich) and cetyltrimethylammonium bromide (CTAB, Sigma-Aldrich) are added to the mixture with additional vigorous stirring for $2 \mathrm{~h}$. The resulting suspension is placed in a Teflon-lined autoclave, and the reactor is deposited inside a microwave oven (MARS model, CEM corporation), which is heated at $190^{\circ} \mathrm{C}$ for $30 \mathrm{~min}$. Upon the completion of the reaction, the resulting precipitate is washed several times with DI water and dried at $120{ }^{\circ} \mathrm{C}$ for $2 \mathrm{~h}$. With the objective of achieving a sintered sample, the dried ceramic powders are uniaxially pressed at $200 \mathrm{MPa}$ in a disk shape. Then, the disk-shaped compact is sintered at $1125{ }^{\circ} \mathrm{C}$ for $2 \mathrm{~h}$ under an air atmosphere, obtaining a density of $4.35 \mathrm{~g}$ $\mathrm{cm}^{-3}$. A standard KNN microstructure is observed with a field emission scanning electron microscope (FE-SEM, Hitachi S-4700), Fig. S2a. $\dagger$ Note that the sintered sample is composed of grains with a platelet morphology, which present an average grain size of $\sim 6.7 \pm 3 \mu \mathrm{m}$; see Fig. S2b. $\dagger$ In addition, one can establish that the domain width $(d)$ can be roughly expressed by the correlation: $d \sim \sqrt{ } a$ (that is, the domain width, $d$, scales as the square root of the grain size, $a),{ }^{6,36}$ meaning that for an average grain size of $a \sim 6.7 \mu \mathrm{m}$ (our case), the $d$ value should be $c a .2 .5 \mu \mathrm{m}$, as shown in Fig. S2c. $\dagger$

In order to analyse the domain structure, the ceramic sample was polished and thermally etched to reveal the topo- 
graphy. With this purpose, the surface of the pellets was carefully polished in two steps. The first step consisted of a "hard polishing" or grinding process using silicon carbide abrasive papers (MetaServ® 250 Grinder-Polisher, Buehler, An ITW Company). This first step was carried out for each pellet in order to obtain parallel surfaces. Finally, in a second step, the surface of the pellet was softly polished with a diamond paste to obtain mirror finish surfaces using a VibroMet® 2 Vibratory Polisher (Buehler, An ITW Company). As a consequence, the micro-roughness is inhibited resulting in an improvement of the sample surface finish. Finally, the ceramic sample was etched at $950{ }^{\circ} \mathrm{C}$ for $5 \mathrm{~min}$ to reveal the grain boundary of the microstructure. The sample was maintained at $T>25{ }^{\circ} \mathrm{C}$ during the confocal Raman microscopy (CRM) measurements using a temperature controller. The identification of the crystal structure (monoclinic symmetry) and orientations of the KNN ceramic were accomplished by means of high-resolution synchrotron X-ray diffraction. Synchrotron radiation X-ray diffraction (SR-XRD) was recorded at the D10B-XRD1 beamline at the National Synchrotron Light Laboratory (LNLS, Campinas, Brazil) on an as-prepared sample mounted on a ceramic sample holder. The X-ray wavelength was set at 1.033200(1) A, and the data were collected in the range $1.208^{\circ} \leq 2 \theta \leq$ $121.180^{\circ}$ with a step length of $0.004^{\circ}$ and a step counting time of $2 \mathrm{~s}$ using a photon energy of $12 \mathrm{keV}$ at room temperature. The refined wavelength using the NIST LaB6 standard reference material was 1.033200(1) A. More information about Rietveld refinement can be found in ESI $1 . \dagger$

\subsection{CRM domain mapping}

The experiments were conducted by confocal Raman microscopy (CRM) coupled with atomic force microscopy (AFM, Witec alpha-300RA) on polished ceramics. Raman spectra were recorded using a $532 \mathrm{~nm}$ excitation laser and a $100 \times$ objective lens $(\mathrm{NA}=0.95)$. The incident laser power was $20 \mathrm{~mW}$. The lateral and vertical resolutions of the confocal microscope were $\sim 250 \mathrm{~nm}$ and $\sim 500 \mathrm{~nm}$, respectively. The spectral resolution of the Raman system was down to $0.02 \mathrm{~cm}^{-1}$. The microscopy sample was mounted on a piezodriven scan platform having $4 \mathrm{~nm}$ lateral and $0.5 \mathrm{~mm}$ vertical positional accuracy. The piezoelectric scanning table allows steps of 3 nanometres ( $0.3 \mathrm{~nm}$ in the vertical direction), giving a very high spatial resolution for confocal Raman microscopy. The microscope base was also fitted with an active vibration isolation system, active $0.7-1000 \mathrm{~Hz}$. The recorded spectra were analyzed by using the Witec Control Plus software.

\subsection{Second harmonic generation (SHG) microscopy}

Spatially resolved SHG experiments were performed on a customized scanning confocal microscope (Olympus BX41) provided with a two-axis XY motorized platform $(0.2 \mu \mathrm{m}$ spatial resolution) driven by the Labspec software. A tuneable femtosecond Ti : sapphire laser (Spectra Physics Model 177-Series), used as a fundamental beam, was focused onto the sample surface by using both the $50 \times$ and $100 \times$ microscope objectives. The SHG signal was collected in backscattering geometry with the same objective and detected with a Peltier cooled photomultiplier tube. The fundamental wavelength was fixed at $800 \mathrm{~nm}$.

\subsection{Angular dependence}

The in situ domain switching was monitored by taking subsequent surface scans at different polarization angles of the same area, using as position reference a significant point of the sample surface visible with the optical microscope. Surface scan Raman images had $5 \mu \mathrm{m}$ of length, $1.5 \mu \mathrm{m}$ of width, $100 \times 30$ spectra of 0.2 seconds of integration time at $20 \mathrm{~mW}$ of laser power. Thus, the Raman image consisting of $100 \times 30$ pixels (3000 spectra) required $\sim 10$ minutes for the acquisition of the whole surface section. Additionally, in order to avoid the photoconductivity effects present in $\mathrm{KNN}$, in our experiments, a polarized light with a wavelength (at $\lambda=532 \mathrm{~nm}$ ) outside the two absorption maxima ${ }^{18,19}$ is chosen and the open circuit configuration is selected. The angle between the polarization of light and the scan direction by using $\Delta \theta=15^{\circ}$ between $\theta=$ $-90^{\circ}$ and $\theta=90^{\circ}$ was rotated. The acquired spectra set was analyzed by using the Witec Control Plus Software.

\subsection{Domain width as a function of the light polarization} angle $(\theta)$

In CRM, in-plane polarized a-domains and out-of-plane polarized c-domains exhibit spectra with unique features. This allows the separation of the signals, and hence, it also helps to determine the domain width evolution as a function of the polarization angle $(\theta)$. For that, the relative volume fraction of each ferroelectric domain was calculated by using the Witec Control Plus software along the different lines for each angle of light polarization on a given sample. Raman spectra for each ferroelectric domain can be compared qualitatively and quantitatively allowing to calculate the domain width $(d)$. By scanning the ferroelectric domain structure and recording the complete Raman spectrum at every spot, we can subsequently filter specific spectral data for a spatially resolved data point and construct both a false-colour $2 \mathrm{D}$ map as well as a linescanned phase volume profile plot of each domain as a function of the $\theta$.

\section{Author contributions}

F. R-M. and J. F. F. designed and performed the experiments, analyzed the results and wrote the manuscript. A. D. C. carried out the Raman image experiments and analyzed the results. R. R-H. and R. U. I. contributed to the structural analysis. L. A. R. and R. P prepared the samples and contributed to the data analysis. M. O. R. and M. L. B. contributed to SHG experiments.

\section{Conflicts of interest}

The authors declare no competing financial interests. 
$1 \quad$ Acknowledgements

This work was supported by the Spanish Ministry of Economy and Competitiveness (MINECO) under the projects MAT201348009-C4-1-P, MAT2013-43301-R and MAT2016-76106-R, the Spanish National Research Council (CSIC) under the project NANOMIND CSIC 201560E068 and the Comunidad de Madrid under the grant S2013/MIT-2740. R. U. I. would like to thank Dr Xavier Turrillas of ICMAB-CSIC (Spain) and Dr Luis. G. Martinez of IPEN-CNEN (Brazil) for their discussions about Rietveld analysis and LNLS for the synchrotron data measurement. F. R.-M. is also indebted to the MINECO for a 'Ramon y Cajal' contract (ref: RyC-2015-18626), which is co-financed by the European Social Fund. L. A. R. and R. P. are also indebted to CONICET, ANPCyT, and the University of Mar del Plata (Argentina) for the funding of this work.

\section{References}

1 K. J. Choi, M. Biegalski, Y. L. Li, A. Sharan, J. Schubert, R. Uecker, P. Reiche, Y. B. Chen, X. Q. Pan, V. Gopalan, L.-Q. Che, D.C. Schlom and C.B. Eom, Science, 2004, 306, 1005-1008.

2 J. Seidel, L. W. Martin, Q. He, Q. Zhan, Y.-H. Chu, A. Rother, M. E. Hawkridge, P. Maksymovych, P. Yu, M. Gajek, N. Balke, S. V. Kalinin, S. Gemming, F. Wang, G. Catalan, J. F. Scott, N. A. Spaldin, J. Orenstein and R. Ramesh, Nat. Mater., 2009, 8, 229-234.

3 L. J. McGilly, P. Yudin, L. Feigl, A. K. Tagantsev and N. Setter, Nat. Nanotechnol., 2015, 10, 145-150.

4 J. C. Agar, A. R. Damodaran, M. B. Okatan, J. Kacher, C. Gammer, R. K. Vasudevan, S. Pandya, L. R. Dedon, R. V. K. Mangalam, G. A. Velarde, S. Jesse, N. Balke, A. M. Minor, S. V. Kalinin and L. W. Martin, Nat. Mater., 2016, 15, 549-556.

5 J. Gonnissen, D. Batuk, G. F. Nataf, L. Jones, A. M. Abakumov, S. Van Aert, D. Schryvers and E. K. H. Salje, Adv. Funct. Mater., 2016, 26, 7599-7604.

6 G. Catalan, J. Seidel, R. Ramesh and J. F. Scott, Rev. Mod. Phys., 2012, 84, 119-156.

7 M. Schröder, A. Haußmann, A. Thiessen, E. Soergel, T. Woike and L. M. Eng, Adv. Funct. Mater., 2012, 22, 39363944.

8 B. S. Kwak, A. Erbil, B. J. Wilkens, J. D. Budai, M. F. Chisholm and L. A. Boatner, Phys. Rev. Lett., 1992, 68, 3733-3736.

9 W. Eerenstein, N. D. Mathur and J. F. Scott, Nature, 2006, 442, 759-765.

10 N. A. Mathur, Nature, 2008, 454, 591-592.

11 N. Liu, R. Dittmer, R. W. Stark and C. Dietz, Nanoscale, 2015, 7, 11787-11796.

12 D. Gobeljic, V. V. Shvartsman, A. Belianinov, B. Okatan, S. Jesse, S. V. Kalinin, C. Groh, J. Rödel and D. C. Lupascu, Nanoscale, 2016, 8, 2168-2176.
13 P. Wang, J. Zhao, L. Wei, Q. Zhu, S. Xie, J. Liu, X. Meng and J. Li, Nanoscale, 2017, 9, 3806-3817.

14 T. Sluka, A. K. Tagantsev, P. Bednyakov and N. Setter, Nat. Commun., 2013, 4, 1808.

15 G. De Luca, M. D. Rossell, J. Schaab, N. Viart, M. Fiebig and M. Trassin, Adv. Mater., 2017, 29, 1605145.

16 F. Rubio-Marcos, A. Del Campo, P. Marchet and J. F. Fernández, Nat. Commun., 2015, 6, 6594.

17 K. Uchino and S. Nomura, Ferroelectrics, 1983, 50, 191-196.

18 K. Uchino, Mater. Res. Innovations, 1997, 1, 163-168.

19 D. Dimos, W. L. Warren, M. B. Sinclair, B. A. Tutle and R. W. Schwartz, J. Appl. Phys., 1994, 76, 4305-4315.

20 V. M. Fridkin, Consultants Bureau, NewYork, 1980.

21 V. M. Fridkin, A. A. Grekov, E. A. Savtchenko and T. R. Volk, J. Phys. Colloq., 1972, 33(C2), 127-129.

22 E. I. Bondarenko, V. Y. Topolov and A. V. Turik, Ferroelectr., Lett. Sect., 1991, 13, 13-19.

23 G. Arlt and N. A. Pertsev, J. Appl. Phys., 1991, 70, 2283.

24 S. Li, A. S. Bhalla, R. E. Newnham and L. E. Cross, Mater. Lett., 1993, 17, 21-26.

25 T. Rojac, H. Ursic, A. Bencan, B. Malic and D. Damjanovic, Adv. Funct. Mater., 2015, 25, 2099-2108.

26 J. Tellier, B. Malic, B. Dkhil, D. Jenko, J. Cilensek and M. Kosec, Solid State Sci., 2009, 11, 320-324.

27 A. S. Everhardt, S. Matzen, N. Domingo, G. Catalan and B. Noheda, Adv. Electron. Mater., 2016, 2, 1500214.

28 F. Rubio-Marcos, A. Del Campo, R. López-Juárez, J. J. Romero and J. F. Fernández, J. Mater. Chem., 2012, 22, 9714-9720.

29 K. Kakimoto, K. Akao, Y. Guo and H. Ohsato, Jpn. J. Appl. Phys., 2005, 44, 7064-7067.

30 F. Rubio-Marcos, M. A. Bañares, J. J. Romero and J. F. Fernández, J. Raman Spectrosc., 2011, 42, 639-643.

31 W. J. Merz, Phys. Rev., 1954, 95, 690-698.

32 E. K. W. Goo, R. K. Mishra and G. Thomas, J. Appl. Phys., 1981, 52, 2940-2943.

33 Z. Kighelman, D. Damjanovic, M. Cantoni and N. Setter, J. Appl. Phys., 2002, 91, 1495-1501.

34 M. Soga, Y. Noguchi, M. Miyayama, H. Okino and T. Yamamoto, Appl. Phys. Lett., 2004, 84, 100-102.

35 Y. L. Li, S. Choudhury, Z. K. Liu and L. Q. Chen, Appl. Phys. Lett., 2003, 83, 1608-1610.

36 G. Arlt, J. Mater. Sci., 1990, 25, 2655-2666.

37 T. Sluka, A. K. Tagantsev, D. Damjanovic, M. Gureev and N. Setter, Nat. Commun., 2012, 3, 748.

38 P. Maksymovych, A. N. Morozovska, P. Yu, E. A. Eliseev, Y.-H. Chu, R. Ramesh, A. P. Baddorf and S. V. Kalinin, Nano Lett., 2012, 12, 209-213.

39 I. Stolichnov, L. Feigl, L. J. McGilly, T. Sluka, X.-K. Wei, E. Colla, A. Crassous, K. Shapovalov, P. Yudin, A. K. Tagantsev and N. Setter, Nano Lett., 2015, 15, 8049-8055.

40 A. Crassous, T. Sluka, A. K. Tagantsev and N. Setter, Nat. Nanotechnol., 2015, 10, 614.

41 A. A. Esin, D. O. Alikin, A. P. Turygin, A. S. Abramov, J. Hreščak, J. Walker, T. Rojac, A. Bencan, B. Malic, A. L. Kholkin and V. Ya. Shur, J. Appl. Phys., 2017, 121, 074101. 
42 R. G. P. McQuaid, M. P. Campbell, R. W. Whatmore, A. Kumar and J. Marty Gregg, Nat. Commun., 2017, 8, 15105.

43 P. S. Bednyakov, T. Sluka, A. K. Tagantsev, D. Damjanovic and N. Setter, Sci. Rep., 2015, 5, 15819.

44 X. Wei, T. Sluka, B. Fraygola, L. Feigl, H. Du, L. Jin, C-L. Jia and N. Setter, ACS Appl. Mater. Interfaces, 2017, 9, 6539-6546.

45 P. Gao, J. Britson, J. R. Jokisaari, C. T. Nelson, S.-H. Baek, Y. Wang, C.-B. Eom, L.-Q. Chen and X. Pan, Nat. Commun., 2013, 4, 2791.
46 H. Deng, H. Zhang, X. Zhao, C. Chen, X. Wang, X. Li, 1 D. Lin, B. Ren, J. Jiao and H. Luo, CrystEngComm, 2015, 17, 2872-2877.

47 T. T. A. Lummen, Y. Gu, J. Wang, S. Lei, F. Xue, A. Kumar, A. T. Barnes, E. Barnes, S. Denev, A. Belianinov, M. Holt, A. N. Morozovska, S. V. Kalinin, L.-Q. Chen and V. Gopalan, Nat. Commun., 2014, 5, 3172.

48 Y. Gu, F. Xue, S. Lei, T. T. A. Lummen, J. Wang, V. Gopalan and L. Q. Chen, Phys. Rev. B: Condens. Matter Mater. Phys, 2014, 90, 024104. 